\title{
A Serum Metabolomic Study Reveals Changes in Metabolites During the Treatment of Lung Cancer-Bearing Mice with Anlotinib
}

\author{
Xiaoting $\operatorname{Pan}^{1,2, *}$ \\ Wenhao Chen ${ }^{2,3, *}$ \\ Mengjun $\mathrm{Nie}^{1,2}$ \\ Yuanjie Liu ${ }^{1,2}$ \\ Zuopeng Xiao ${ }^{1,2}$ \\ Ying Zhang ${ }^{1,2}$ \\ Wei Zhang' \\ Xi Zou'
}

'The Affiliated Hospital of Nanjing University of Chinese Medicine, Jiangsu Province Hospital of Chinese Medicine, Nanjing, Jiangsu, 210029, People's Republic of China; ${ }^{2}$ No.I Clinical Medical College, Nanjing University of Chinese Medicine, Nanjing, Jiangsu, 210023 , People's Republic of China; ${ }^{3}$ jiangsu Cancer Hospital, Nanjing, Jiangsu,

210009, People's Republic of China

*These authors contributed equally to this work
Correspondence: Xi Zou

The Affiliated Hospital of Nanjing

University of Chinese Medicine, Jiangsu

Province Hospital of Chinese Medicine,

Nanjing, Jiangsu, 210029, People's

Republic of China

Tel +86-18051983568

Email zxvery@I26.com
Background: Anlotinib is a vascular endothelial growth factor receptor tyrosine kinase inhibitor recommended for the treatment of advanced lung cancer patients after at least two previous systemic chemotherapies. Currently, many patients with lung cancer do not respond well to anlotinib treatment. Therefore, the aim of this metabolomic study was to determine the internal mechanism of anlotinib action at the molecular level and to identify the potential biomarkers and pathways associated with the therapeutic effects of anlotinib.

Methods: A total of 20 male nude mice were randomly divided into 2 groups and treated with anlotinib or physiological saline. Ultra-high-performance liquid chromatographyquadrupole time-of-flight mass spectrometry was performed to analyze the serum samples and determine the differential metabolites and pathways between anlotinib and control groups.

Results: We observed significant differences between the anlotinib and control groups, and 13 endogenous differential metabolites and 5 potential metabolic pathways were identified. Glyoxylate and dicarboxylate metabolism, tryptophan metabolism, glycine, serine and threonine metabolism, phenylalanine metabolism and valine, leucine and isoleucine biosynthesis were the most important pathways regulated by anlotinib in vivo. Notably, these 5 differential pathways were highly associated with the TCA cycle, which is important in the proliferation and apoptosis of cancer cells.

Conclusion: This serum metabolomic study revealed distinct metabolic profiles in lung cancer-bearing mice treated with anlotinib and identified differential metabolites and pathways between the anlotinib and control groups, which may provide new ideas for the clinical application of anlotinib.

Keywords: anlotinib, lung cancer, LC-MS, differential metabolites, pathway

\section{Introduction}

Lung cancer is one of the most commonly diagnosed malignant tumors with the highest morbidity and mortality worldwide, and its incidence and mortality among all cancer cases have risen to $11.6 \%$ and $18.4 \%$, respectively. ${ }^{1}$ Notably, Micronesia/ Polynesia, Eastern Europe and Eastern Asia are high-risk regions for lung cancer, especially China. ${ }^{1}$ A retrospective survey found that over the past 40 years, the proportion of lung cancer in cancer deaths in China has risen from $7.35 \%$ to $22.70 \%$, leaping from 5 th to $1 \mathrm{st}^{2}{ }^{2}$ Due to the insidious onset of lung cancer, most patients are already in an advanced stage when they seek medical care. Consequently, they lose the opportunity for surgical treatment, and the response 
rate to standard chemotherapy in advanced stages is only $15-30 \% .^{3}$ In this case, targeted therapy plays a significant role in the treatment of lung cancer and can benefit a large number of lung cancer patients in the clinic.

Anlotinib is a novel oral tyrosine kinase inhibitor targeting VEGFR1, VEGFR2/KDR, VEGFR3, c-Kit, PDGFR- $\alpha$ and fibroblast growth factor receptors (FGFR1, FGFR2 and FGFR3) that simultaneously inhibits the proliferation and angiogenesis of cancer cells. ${ }^{4,5}$ A Phase III clinical experiment of non-small cell lung cancer (Clinical Trail Registry ID: NCT 02388919) revealed that compared to placebo, anlotinib significantly improved overall survival and quality of life $\mathrm{C}^{6,7}$ and has been approved in China for third-line treatment of advanced non-small cell lung cancer (NSCLC). However, there are still many NSCLC patients who are administered anlotinib in practice and have unsatisfactory curative outcomes. In other words, only a fraction of patients with lung cancer benefit from anlotinib treatment. Therefore, it is urgent to identify appropriate biomarkers related to the therapeutic effects of anlotinib and screen effective patient groups.

To address this issue, metabolomics, a discipline developed following genomics and proteomics, were used to identify the key metabolites in lung cancer-bearing mice after administration of anlotinib. To date, this method has been increasingly applied to the discovery of new candidate biomarkers for cancer diagnosis, drug reaction and toxicity. ${ }^{8}$ In addition, compared to nuclear magnetic resonance (NMR) and gas chromatography-mass spectrometry (GC-MS), liquid chromatography-mass spectrometry (LCMS) technology has higher sensitivity than $\mathrm{NMR}^{4}$ and does not require complicated derivatization pretreatments, such as GC-MS; hence, it is suitable for pharmacodynamic metabolomics research. Over recent decades, researchers have primarily focused on identifying potential biomarkers for the early diagnosis in lung cancer. For instance, Mo et al described the small molecule metabolites of lung adenocarcinoma patients using LC-MS technology and revealed that 14 differential metabolites, including adenosine 3-monophosphate, glycerol and creatine, differed from benign lung cancer patients. ${ }^{9}$ Zhou et al used LCMS/MS combined with iTRAQ8-plex labeling technology to screen out potential early diagnosis and metastasis biomarkers for lung adenocarcinoma. They found that the levels of elongation factor 1-alpha 2, proteasome subunit alpha type and spermatogenesis-associated protein were increased in the serum of lung cancer patients. The levels of transmembrane protein 143 , cadherin 5 , fibronectin 1 , and collectin-11 were decreased in the serum of patients with metastases compared to those of nonmetastatic lung cancer patients. ${ }^{10}$ Pamungkas et al found that bisphenol A, retinol and L-proline, which are associated with the development of lung cancer, might be new diagnostic tools in lung cancer. ${ }^{11}$ To date, the application of metabolomics in lung cancer has also been used to analyze the efficacy of antineoplastic drugs. For example, Ma et al conducted cell metabolomics to explore the potential mechanism of acquired resistance to osimertinib, demonstrating that the regulation of amino acid metabolism, the energy supply process and the PI3K-Akt and HIF-1 signaling pathways were highly related to osimertinib resistance. ${ }^{12}$ The HIF-1 signaling pathway, which modulates P-glycoprotein expression, the PI3K-Akt pathway, which regulates survivin expression, and oxidative phosphorylation were all upregulated, while arginine and proline metabolism, which regulates NO production and glycolysis/gluconeogenesis, were downregulated in osimertinib resistance. Peng et al examined 43 lung cancer patients with different responses to cisplatin to explore the mechanisms of cisplatin resistance by metabolomics and screened 8 novel biomarkers significantly associated with the platinum chemotherapy response, and a prediction model with these biomarkers was employed to predict chemotherapy response in a validation set, exhibiting $100 \%$ sensitivity and specificity. ${ }^{13}$ All of these research practices indicate that metabolomics has high application value in understanding the specific changes in metabolites in lung cancer patients after drug action, and it is expected to clarify the drug effect characteristics and advantages of drugs for treating lung cancer and their internal mechanism of action at the molecular level.

\section{Materials and Methods \\ Chemicals and Reagents}

Anlotinib hydrochloride capsules (12 mg/capsule) were obtained from Zhengda Tianqing Company (batch number: H20180004). HPLC-grade methanol, ammonium hydroxide, ammonium acetate and acetonitrile were purchased from CNW Technologies. The internal standard, 2-chloro-L-phenylalanine, was obtained from Shanghai Hengbai Biotech Co., Ltd.

\section{Experimental Animals}

Twenty male nude mice weighing $18-22 \mathrm{~g}$ at the age of 4 weeks, were purchased from Nanjing Branch of Beijing 
Vittorio Laboratory Animal Technology Co., Ltd. and were fed standard chow and adapted to the environment for 1 week before modeling. Human lung cancer A549 cells, obtained from the Central Laboratory of Jiangsu Province Hospital of Traditional Chinese Medicine, were cultured during the growth phase and subsequently collected. It was approved by the Ethics Committee of the Affiliated Hospital of Nanjing University of Chinese Medicine (2018 DW-08-03) and obeyed the principles of "Guide for the Care and Use of Laboratory Animals" during the experiment. The density of A549 cells was adjusted to $1.5^{*} 10^{7} / \mathrm{mL}$, and each mouse was injected with $0.2 \mathrm{~mL}$ A549 cell suspension into the right axilla. Once the tumor reached a diameter of 5-7 mm, 20 mice were randomly divided into 2 groups: the control group and the anlotinib group. The control group $(n=10)$ was administered $0.1 \mathrm{~mL} / 10 \mathrm{~g}$ physiological saline once per day by gavage for 14 days, while the anlotinib group $(\mathrm{n}=10)$ received $1.5 \mathrm{mg} / \mathrm{kg}$ or $0.1 \mathrm{~mL} / 10 \mathrm{~g}$ anlotinib dissolved in physiological saline daily for 14 days via gavage. All mice were housed in an SPF environment at Nanjing University of Traditional Chinese Medicine at a temperature of $26-28^{\circ} \mathrm{C}$, relative humidity of $40-60 \%$ and a natural light/dark cycle. This study was approved by the Ethics Committee of the Affiliated Hospital of Nanjing University of Chinese Medicine (2018 DW-08-03) and it was conducted in accordance with the requirements of "Guide for the Care and Use of Laboratory Animals".

\section{Sample Collection}

After the last administration, blood samples were collected from the inner canthus vein of mice in each group and kept in a vacutainer tube for $1 \mathrm{~h}$. Then, we centrifuged the clotted blood samples at $3000 \mathrm{rpm}$ for $10 \mathrm{~min}$ and collected the serum from the supernatant. Before the LC-MS analysis was conducted, all serum samples were stored at $-80^{\circ} \mathrm{C}$.

Each $100 \mu \mathrm{L}$ of serum sample was mixed with $400 \mu \mathrm{L}$ extract solution (acetonitrile:methanol=1:1 (v/v)) containing internal standard (2-chloro-L-phenylalanine, $2 \mathrm{~g} / \mathrm{mL}$ ), vortexed for $30 \mathrm{~s}$ and sonicated for $10 \mathrm{~min}$ in an ice-water bath. After standing at $-40^{\circ} \mathrm{C}$ for $1 \mathrm{~h}$, the serum samples were centrifuged at $4^{\circ} \mathrm{C}$ for $15 \mathrm{~min}$ at $10,000 \mathrm{rpm}$, and then $425 \mu \mathrm{L}$ of the supernatant was collected and dried. Then, $200 \mu \mathrm{L}$ of $50 \%$ acetonitrile was added to the dried samples for reconstitution by sonication on ice for $10 \mathrm{~min}$. Next, the solution was centrifuged at $13,000 \mathrm{rpm}$ for 15 min at $4^{\circ} \mathrm{C}$, and $75 \mu \mathrm{L}$ of supernatant was used for LC-MS analysis. To determine system stability, equal aliquots of the supernatants from each sample were pooled and mixed to prepare QC samples.

\section{LC-MS/MS Analysis}

UHPLC separation was performed using a 1290 Infinity series UHPLC System (Agilent Technologies) with a UPLC BEH amide column $\left(2.1^{*} 100 \mathrm{~mm}, 1.7 \mu \mathrm{m}\right.$, Waters). The column temperature was maintained at $25^{\circ} \mathrm{C}$. The mobile phase was A, containing $25 \mathrm{mmol} / \mathrm{L}$ ammonium acetate and $25 \mathrm{mmol} / \mathrm{L}$ ammonia hydroxide $(\mathrm{pH}=9.75)$ in water, and $\mathrm{B}$ was acetonitrile. The gradient conditions were as follows: $95 \% \mathrm{~B}$ at $0-0.5 \mathrm{~min}, 95-65 \% \mathrm{~B}$ at $0.5-7.0 \mathrm{~min}$, $65-40 \% \mathrm{~B}$ at $7.0-8.0 \mathrm{~min}, 40 \% \mathrm{~B}$ at $8.0-9.0 \mathrm{~min}, 40-95 \%$ $\mathrm{B}$ at $9.0-9.1 \mathrm{~min}$, and $95 \% \mathrm{~B}$ at $9.1-12.0 \mathrm{~min}$. The flow rate of the mobile phase was $0.5 \mathrm{~L} / \mathrm{min}$, and the autosampler temperature was set at $4^{\circ} \mathrm{C}$. The injection volume was $1 \mu \mathrm{L}$ for both negative mode and positive mode.

During the LC/MS experiment, TripleTOF 6600 mass spectrometry (AB Sciex) and information-dependent acquisition (IDA) were adapted to acquire MS/MS spectra. In IDA mode, Analyst TF (version 1.7, AB Sciex), the acquisition software, evaluated the full scan survey MS data and collected the MS/MS spectra depending on the preselected criteria. The 12 most intensive precursor ions with intensities greater than 100 were selected for MS/MS in each cycle; the collision energy was $30 \mathrm{eV}$, and the cycle time was $0.56 \mathrm{~s}$. The ESI source parameters were set as follows: gas 1:60 psi; gas 2: 60 psi; CUR: 35 psi; TEM: $600^{\circ} \mathrm{C}$; DP: $60 \mathrm{~V}$; ISVF: $5000 \mathrm{~V}$ in positive mode and $-4000 \mathrm{~V}$ in negative mode.

\section{Data Processing and Statistical Analysis}

The raw LC-MS data files were converted to the mzXML format by Proteo Wizard. Peak deconvolution, alignment and integration were processed using the $\mathrm{R}$ package XCMS (version 3.2). Minifract was set to 0.5 , and the cutoff was set as 0.6. In addition, an in-house MS2 database was used to identify the metabolites. A $t$-test was used to analyze univariate data, and principal component analysis (PCA) and orthogonal partial least squares discrimination analysis (OPLS-DA) were used to analyze multivariate data.

\section{Results}

\section{Metabolic Profiles of Serum Samples}

UHPLC-Q-TOF/MS was used to analyze serum samples collected from lung cancer-bearing mice with or without 
anlotinib treatment, and 2330 peaks in positive ion mode and 1194 peaks in negative ion mode were identified. After data management, including removing the noise of each peak, missing value recording, normalization based on internal standards, 3405 peaks, including 2260 peaks in positive ion mode and 1145 peaks in negative ion mode, were reserved for further multivariate statistical analysis. Typical total ion chromatograms (TICs) of metabolic profiles for the anlotinib and control groups are shown in Figure 1.

\section{Multivariate Statistical Analysis}

An unsupervised analysis was applied to the metabolic profiling to visualize the distribution of anlotinib and the control group. The PCA score plots of global views obtained from these two groups are provided in Figure 2A. The anlotinib group and control group were clearly distinguished from each other. Each point represents one sample, and all of the samples were in the $95 \%$ Hotelling's T-squared ellipse. To avoid the invalid supervision of PCA models and maximize the differences
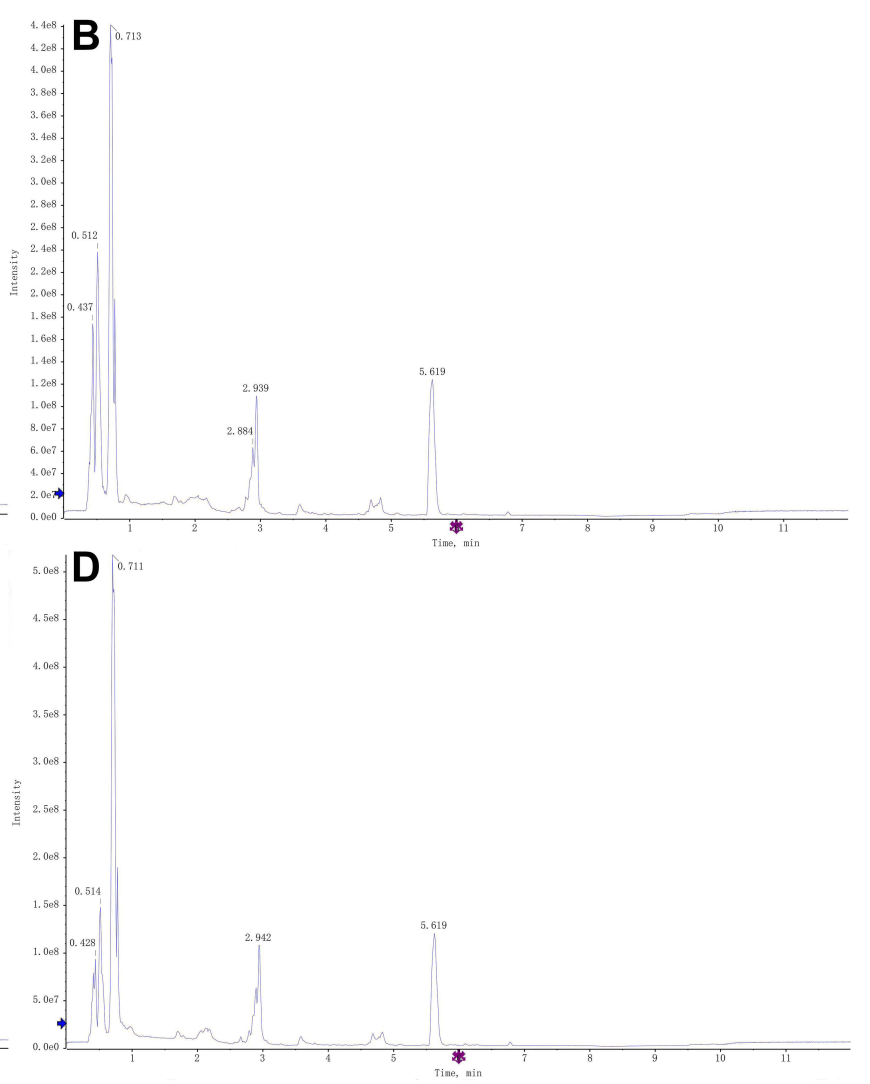

Figure I Typical TICs of metabolic profiles in the anlotinib and control groups. (A) The anlotinib group in positive ion mode. (B) The anlotinib group in negative ion mode. (C) The control group in positive ion mode. (D) The control group in negative ion mode.
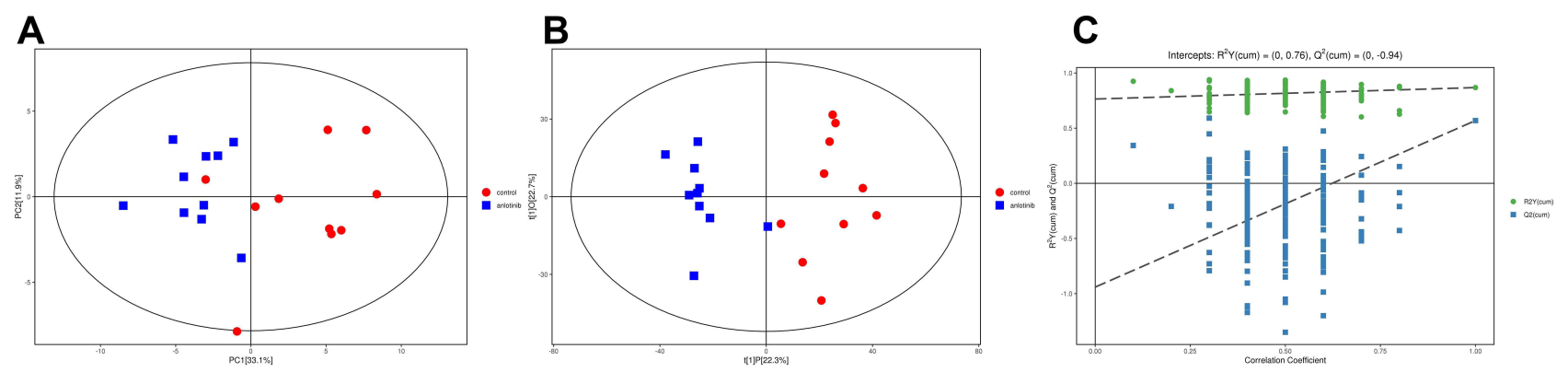

Figure 2 (A) PCA score plot and (B) OPLS-DA score plot between the anlotinib and control groups. The blue points represent the samples in the anlotinib group, and the red points represent the samples in the control group. (C) The 200 permutation test. 
between groups, the OPLS-DA supervised pattern recognition method and the permutation test (Figure 2C) were employed. As expected, remarkable separation was observed (Figure 2B), suggesting that there were differential metabolites between the two groups. The parameters of OPLS-DA were $\mathrm{R}^{2} \mathrm{X}=0.45, \mathrm{R}^{2} \mathrm{Y}=0.868$, and $\mathrm{Q}^{2}=0.569$.

\section{Differences in Metabolites and Related Pathways Between the Anlotinib and Control Groups}

To identify the potential targets of anlotinib, differential metabolites were carefully screened based on PCA and OPLS-DA modeling analysis. In total, with reference to the Human Metabolome Database (HMDB) and KEGG, 73 differential metabolites were identified according to VIP $>1$ and $\mathrm{P}<0.05$ criteria. Finally, of all 73 metabolites, we selected the 13 endogenous differential metabolites with FC>1.5 (Table 1).

Metabolite set enrichment analysis was used to determine the differential metabolic pathways, and the most related pathways were glyoxylate and dicarboxylate metabolism; tryptophan metabolism; glycine, serine and threonine metabolism; phenylalanine metabolism; and valine, leucine and isoleucine biosynthesis (Figure 3).

\section{Discussion}

In this study, LC-MS was used to determine the changes in metabolites upon treatment of lung cancer-bearing mice with anlotinib. First, the metabolic profile of lung cancer-

Table I Metabolites and Their Related Metabolic Pathways in the Anlotinib and Control Groups

\begin{tabular}{|l|c|c|c|c|}
\hline No. & Metabolites & $\begin{array}{c}\text { VIP } \\
\text { value }\end{array}$ & P-value & $\begin{array}{c}\text { Fold } \\
\text { Change }\end{array}$ \\
\hline 1 & Myristic acid & 1.529 & 0.001 & 1.551 \\
2 & Cytidine & 1.551 & 0.001 & 3.187 \\
3 & Indole & 1.575 & 0.003 & 3.865 \\
4 & Deoxycytidine & 1.377 & 0.000 & 2.958 \\
5 & Dimethylglycine & 1.205 & 0.001 & 1.854 \\
6 & Cytosine & 1.314 & 0.000 & 2.117 \\
7 & Tetracosanoic acid & 1.456 & 0.003 & 1.710 \\
8 & Behenic acid & 1.393 & 0.003 & 1.742 \\
9 & L-Gulonic gamma- & 1.429 & 0.003 & 1.537 \\
& lactone & & & \\
10 & Glycyl-L-leucine & 1.466 & 0.017 & 1.791 \\
II & 6-Hydroxymelatonin & 1.484 & 0.001 & 2.858 \\
12 & N-Formylmethionine & 1.486 & 0.008 & 2.307 \\
13 & Glutathione disulfide & 1.499 & 0.008 & 1.778 \\
\hline
\end{tabular}

Notes: All values retain 3 decimal places. bearing mice in response to different treatments was obtained from LC-MS analysis, and a total of 3405 peaks were observed. After removing features with greater than $20 \%$ missing values, comparing them to the databases and based on the VIP values, P-values and FC values, 13 endogenous differential metabolites were finally screened out. Then, we investigated the differential metabolic pathways according to all differential metabolites. Glyoxylate and dicarboxylate metabolism, tryptophan metabolism, glycine, serine and threonine metabolism, phenylalanine metabolism and valine, leucine and isoleucine biosynthesis were identified as potential pathways affected by anlotinib.

\section{Glyoxylate and Dicarboxylate Metabolism}

The glyoxylate shunt is an anaplerotic pathway of the tricarboxylic acid cycle (TCA), which can provide carbon skeletons for biomass production and gluconeogenesis. $^{14,15}$ In other words, it is significantly related to glucose metabolism. Mounting evidence supports the supposition that metabolic reprogramming plays an important role in the progression and carcinogenesis of cancer. $^{16,17}$ For example, Chen et al found that low-folate stress reprogrammed metabostemness signatures through activating the mTOR signaling pathway to promote metastasis and tumorigenicity in lung cancer. ${ }^{18} \mathrm{Fu}$ et al revealed that mitochondria slows tumor growth and increases the survival of animals by injecting mitochondria isolated from healthy mouse livers into a melanoma mouse model with lung metastasis. The antitumor effect of mitochondria was associated by interfering with tumor cell metabolism, such as reducing glycolysis and producing an oxidative intracellular environment. ${ }^{19}$ In addition, in our study, we found that levels of intermediates in glucose metabolism, including isocitrate, citrate, and cis-aconitate, were decreased in response to anlotinib treatment, indicating that anlotinib may exert an antitumor effect by regulating glyoxylate and dicarboxylate metabolism.

\section{Tryptophan Metabolism}

Tryptophan is an amino acid essential for protein synthesis and a precursor of many bioactive compounds. Due to its antitumor immune responses and effects on tumor cell intrinsic malignant properties, it is a critical regulator of cancer progression. ${ }^{20}$ It has been reported that depletion of tryptophan facilitates tumor immune escape ${ }^{21}$ and that the metabolites of free tryptophan metabolized through the kynurenine pathway have crucial functions in immune response regulation and neurotransmission. ${ }^{22}$ Based on 


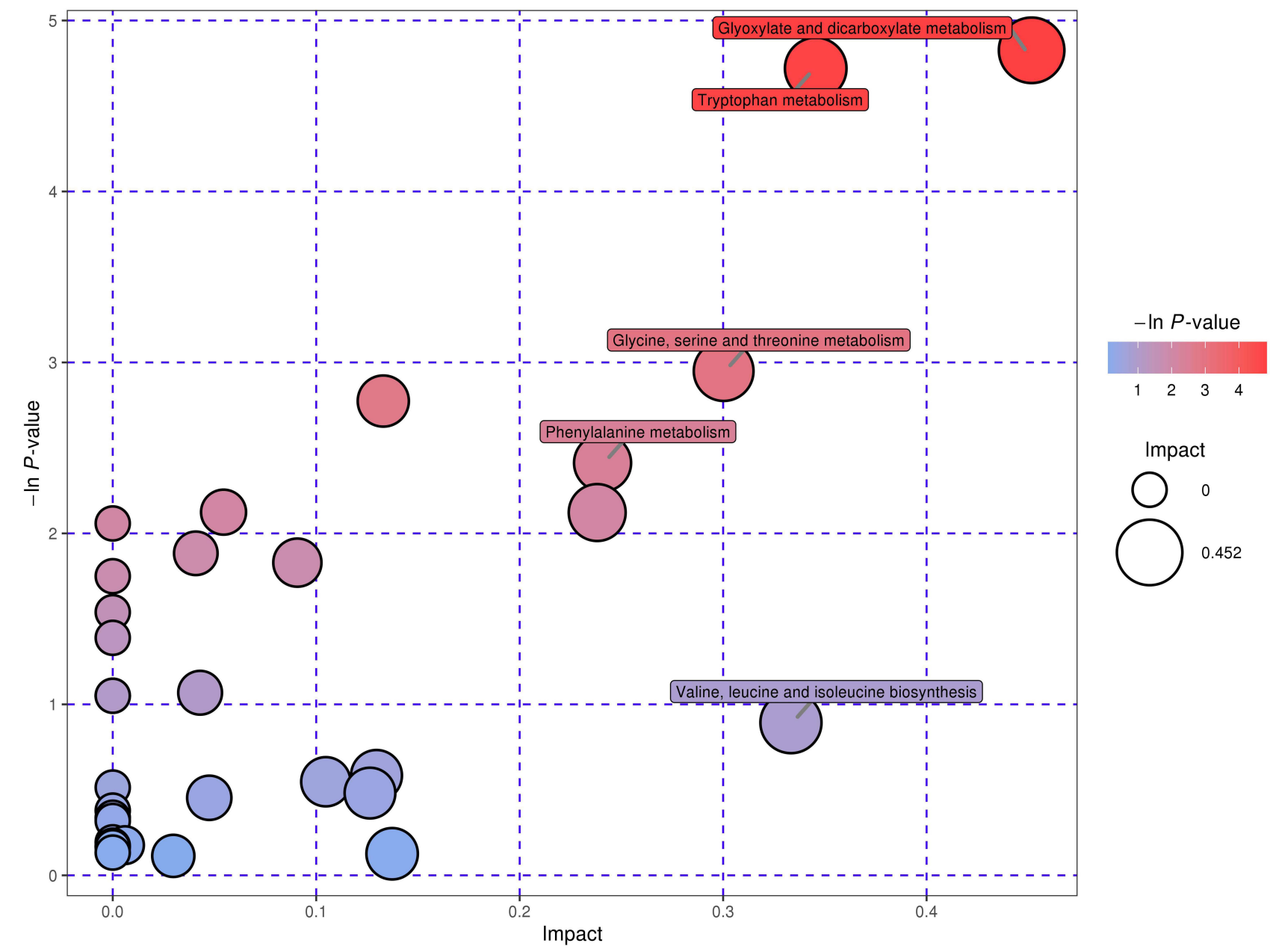

Figure 3 Pathway enrichment analysis and summary of pathway impact based on KEGG pathway networks.

the mechanism mentioned above, several small-molecule inhibitors targeting tryptophan catabolism have been developed and tested in clinical trials. ${ }^{23,24}$ Furthermore, we found that after anlotinib treatment, levels of tryptophan were also increased, suggesting an important role of anlotinib in tumor immune escape.

\section{Glycine, Serine and Threonine Metabolism}

Accumulating evidence has shown that many cancers exhibit a surging demand for specific amino acids, such as serine, and depend on exogenous supply or upregulated endogenous synthesis. ${ }^{25,26}$ Serine, synthesized from 3-phosphoglycerate in the glycolytic pathway, can be used for the biosynthesis of many other intermediates, such as glycine, threonine metabolism, proteins, pyrimidines, purines and sphingolipids. ${ }^{27}$ In addition, it was reported that in breast cancer with high expression of phosphoglycerate dehydrogenase, the proportion of serine synthesis in total replenishments for TCA cycle intermediates was up to $50 \%$ by virtue of converting glutamate into 2-oxoglutarate. ${ }^{27}$ Based on these findings, we inferred that glycine, serine and threonine metabolism was significantly associated with the TCA cycle and the Warburg effect in cancer cells. Additionally, over the past decade, it has been well documented that glycine is highly correlated with the proliferation of cancer cells ${ }^{28}$ and an essential amino acid for the synthesis of glutathione, which can scavenge reactive species, maintain the stability of the intracellular redox state, regulate the immune system and remove carcinogens. ${ }^{29,30}$ Therefore, increasing attention has been given to the development of antitumor drugs targeting glycine metabolism. According to the report of $\mathrm{Hu}$ and coworkers, glycodeoxycholic acid and glycocholic acid might be potential biomarkers for the efficacy of anlotinib, and both of them are associated with glycine. ${ }^{31}$ Guo et al found that after treatment with afatinib, gefitinib and 
Targretin, the metabolism of glycine, serine and threonine was significantly disturbed, ${ }^{32}$ suggesting that this metabolic pathway might be relevant to the underlying mechanism of many antitumor agents. In our study, anlotinib also affected glycine, serine and threonine metabolism, which further supports this point.

\section{Phenylalanine Metabolism}

Phenylalanine is one of the essential amino acids in the human diet and is necessary for the production of other nonessential amino acids. The rapid proliferation of cancer cells requires a large amount of nutrients, such as amino acids, leading to significant differences in the concentration of free amino acids. A previous study showed that phenylalanine and citrulline are the most robust and striking features of NSCLC in blood. ${ }^{33}$ Additionally, it was reported that the activity of phenylalanine hydroxylase, which converts phenylalanine to tyrosine, is dysfunctional in inflammatory and malignant disease states. ${ }^{34,35}$

\section{Valine, Leucine and Isoleucine Biosynthesis}

Valine, leucine and isoleucine are branched-chain amino acids (BCAAs) that play critical roles in protein synthesis, glucose metabolism and nutrition metabolism pathways, such as the mammalian target of rapamycin (mTOR) and phosphoinositide 3-kinase-protein kinase (PI3K-AKT) pathways. $^{36,37}$ Studies have illustrated that amino acids can activate AKT signaling through class I PI3K and mTOR, affecting the growth, proliferation and survival of cancer cells. ${ }^{38}$ A previous study also showed that reprogrammed BCAA metabolism drives cancer progression and regulates cancer development directly. ${ }^{39}$ Therefore, the regulation of valine, leucine and isoleucine biosynthesis may be one of the underlying mechanisms of the antitumor effects of anlotinib at the molecular level.

\section{Conclusions}

In this study, we found that the metabolic profiles of serum samples in the anlotinib group differed from those of the control group. In addition, 13 differential metabolites and 5 significantly altered pathways were identified. Anlotinib significantly regulates glyoxylate and dicarboxylate metabolism, tryptophan metabolism, glycine, serine and threonine metabolism, phenylalanine metabolism and valine, leucine and isoleucine biosynthesis to exert its antitumor effects in vivo. Of note, the 5 differential pathways were highly associated with the TCA cycle, which is important for the proliferation and apoptosis of cancer cells. This study elucidated the potential metabolic pathways that anlotinib may affect in vivo, providing new ideas for clinical medication. Moreover, in future studies, we will verify the differentiated metabolites and pathways screened in this study through targeted metabolomics, determining the most related metabolites and metabolic pathways to the efficacy of anlotinib. Next, we will also try to set up a prediction model using these potential biomarkers to predict the response to anlotinib in practice to benefit more patients.

\section{Abbreviations}

NMR, nuclear magnetic resonance; GC-MS, gas chromatography-mass spectrometry; LC-MS, liquid chromatography-mass spectrometry; TICs, Total ion chromatograms; TCA, tricarboxylic acid cycle; BCAAs, branched-chain amino acids; NSCLC, non-small cell lung cancer.

\section{Acknowledgments}

We appreciate the support of the Beijing Medical and Health Foundation (YWJKJJHKYJJ-F2012E). Xiaoting Pan and Wenhao Chen are the co-first authors and devote equally to this article.

\section{Disclosure}

The authors report no conflicts of interest in this work.

\section{References}

1. Bray F, Ferlay J, Soerjomataram I, Siegel RL, Torre LA, Jemal A. Global cancer statistics 2018: GLOBOCAN estimates of incidence and mortality worldwide for 36 cancers in 185 countries. CA Cancer J Clin. 2018;68(6):394-424. doi:10.3322/caac.21492

2. Chen W, Zhang S, Zou X. Estimation and projection of lung cancer incidence and mortality in China. Chin J Lung Cancer. 2010;13:488-493.

3. Lu S, Yu Y, Yang Y. Retrospect and prospect for lung cancer in China: clinical advances of immune checkpoint inhibitors. Oncologist. 2019;24(S1):S21-S30. doi:10.1634/theoncologist.2019-IO-S1-s02

4. Xie C, Wan X, Quan H, et al. Preclinical characterization of anlotinib, a highly potent and selective vascular endothelial growth factor receptor-2 inhibitor. Cancer Sci. 2018;109(4):1207-1219.

5. Sebastien T, Chieh-Hsiang Y, Maria R, et al. Abstract 3244:treatment of endometrial cancer cells with a new small tyrosine kinase inhibitor targeting mutated fibroblast growth factor receptor-2. Cancer Res. 2017;77:3244.

6. Han B, Li K, Wang Q, et al. Effect of anlotinib as a third-line or further treatment on overall survival of patients with advanced non-small cell lung cancer: the ALTER 0303 Phase 3 randomized clinical trial. JAMA Oncol. 2018;4(11):1569-1575. doi:10.1001/jamaoncol.2018.3039

7. Si X, Zhang L, Wang $\mathrm{H}$, et al. Quality of life results from a randomized, double-blinded, placebo-controlled, multi-center phase III trial of anlotinib in patients with advanced non-small cell lung cancer. Lung Cancer. 2018;122:32-37. doi:10.1016/j.lungcan.2018. 05.013 
8. Miolo G, Muraro E, Caruso D, et al. Pharmacometabolomics study identifies circulating spermidine and tryptophan as potential biomarkers associated with the complete pathological response to trastuzumab-paclitaxel neoadjuvant therapy in HER-2 positive breast cancer. Oncotarget. 2016;7(26):39809-39822. doi:10.18632/ oncotarget.9489

9. Mo L, Wei B, Liang R, et al. Exploring potential biomarkers for lung adenocarcinoma using LC-MS/MS metabolomics. J Int Med Res. 2020;48(4):300060519897215. doi:10.1177/0300060519897215

10. Zhou M, Kong Y, Wang X, et al. LC-MS/MS-Based quantitative proteomics analysis of different stages of non-small-cell lung cancer. Biomed Res Int. 2021;2021:5561569.

11. Pamungkas AD, Park C, Lee S, Jee SH, Park YH. High resolution metabolomics to discriminate compounds in serum of male lung cancer patients in South Korea. Respir Res. 2016;17(1):100. doi:10.1186/s12931-016-0419-3

12. Ma Q, Wang J, Ren Y, Meng F, Zeng L. Pathological mechanistic studies of Osimertinib resistance in non-small-cell lung cancer cells using an integrative metabolomics-proteomics analysis. J Oncol. 2020;2020:6249829.

13. Feng P, Yingzi L, Chenjie H, et al. Prediction of platinum-based chemotherapy efficacy in lung cancer based on LC-MS metabolomics approach. J Pharm Biomed Anal. 2018;154:95-101. doi:10.1016/j. jpba.2018.02.051

14. Crousilles A, Dolan SK, Brear P, Chirgadze DY, Welch M. Gluconeogenic precursor availability regulates flux through the glyoxylate shunt in Pseudomonas aeruginosa. $J$ Biol Chem. 2018;293(37):14260-14269. doi:10.1074/jbc.RA118.004514

15. Deng Y, Ma N, Zhu K, Mao Y, Wei X, Zhao Y. Balancing the carbon flux distributions between the TCA cycle and glyoxylate shunt to produce glycolate at high yield and titer in Escherichia coli. Metab Eng. 2018;46:28-34. doi:10.1016/j.ymben.2018.02.008

16. Kota A, Mai CL, Melissa Z, et al. Metabolic profiling of formalin-fixed paraffin-embedded tissues discriminates normal colon from colorectal cancer. Mol Cancer Res. 2020;18(6):883-890. doi:10.1158/1541-7786.MCR-19-1091

17. Tong Y, Gao WQ, Liu YF. Metabolic heterogeneity in cancer: an overview and therapeutic implications. Biochim Biophys Acta Rev Cancer. 2020;1874(2):188421. doi:10.1016/j.bbcan.2020.188421

18. Chen WJ, Huang RFS. Low-folate stress reprograms cancer stem cell-like potentials and bioenergetics metabolism through activation of mTOR signaling pathway to promote in vitro invasion and in vivo tumorigenicity of lung cancers. J Nutr Biochem. 2018;53:28-38. doi:10.1016/j.jnutbio.2017.10.001

19. Fu A, Hou Y, Yu Z, et al. Healthy mitochondria inhibit the metastatic melanoma in lungs. Int Biol Sci. 2019;15(12):2707-2718. doi:10.7150/ijbs.38104

20. Christiane AO, Luis FSP, Soumya RM, et al. The therapeutic potential of targeting tryptophan catabolism in cancer. $\mathrm{Br} J$ Cancer. 2020;122(1):30-44. doi:10.1038/s41416-019-0664-6

21. Brenk M, Scheler M, Koch S, et al. Tryptophan deprivation induces inhibitory receptors ILT3 and ILT4 on dendritic cells favoring the induction of human $\mathrm{CD} 4+\mathrm{CD} 25+\mathrm{Foxp} 3+\mathrm{T}$ regulatory cells. J Immunol. 2009;183(1):145-154. doi:10.4049/jimmunol.0803277

22. Platten M, Nollen EAA, Rohrig UF, Fallarino F, Opitz C. Tryptophan metabolism as a common therapeutic target in cancer, neurodegeneration and beyond. Nat Rev Drug Discov. 2019;18:379-401.
23. Cheong JE, Ekkati A, Sun L. A patent review of IDO1 inhibitors for cancer. Expert Opin Ther Pat. 2018;28(4):317-330. doi:10.1080/ 13543776.2018.1441290

24. Andersen MH. The targeting of tumor-associated macrophages by vaccination. Cell Stress. 2019;3(5):139-140. doi:10.15698/ cst2019.05.185

25. Lee N, Kim D. Cancer metabolism: fueling more than just growth. Mol Cells. 2016;39(12):847-854. doi:10.14348/molcells.2016.0310

26. Mattaini KR, Sullivan MR, Heiden MGV. The importance of serine metabolism in cancer. $J$ Cell Biol. 2016;214(3):249-257. doi:10.1083/jcb.201604085

27. Possemato R, Marks KM, Shaul YD, et al. Functional genomics reveal that the serine synthesis pathway is essential in breast cancer. Nature. 2011;476(7360):345-350. doi:10.1038/nature10350

28. Redalen KR, Sitter B, Bathen TF, et al. High tumor glycine concentration is an adverse prognostic factor in locally advanced rectal cancer. Radiother Oncol. 2016;118(2):393-398. doi:10.1016/j. radonc.2015.11.031

29. Liu C, Liu H, Li Y, et al. Intracellular glutathione content influences the sensitivity of lung cancer cell lines to methylseleninic acid. Mol Carcinog. 2012;51(4):303-314. doi:10.1002/mc.20781

30. Kennedy L, Sandhu JK, Harper ME, Cuperlovic-Culf M. Role of glutathione in cancer: from mechanisms to therapies. Biomolecules. 2020;10(10):1429. doi:10.3390/biom10101429

31. Hu T, An Z, Sun Y, et al. Longitudinal pharmacometabonomics for predicting malignant tumor patients responses to anlotinib therapy: phenotype, efficacy, and toxicity. Front Oncol. 2020;10:548300. doi:10.3389/fonc. 2020.548300

32. Guo K, Cao Y, Li Z, et al. Glycine metabolomic changes induced by anticancer agents in A549 cells. Amino Acids. 2020;52(5):793-809. doi:10.1007/s00726-020-02853-0

33. Klupczynska A, Derezinski P, Dyszkiewicz W, Pawlak K, Kasprzyk M, Kokot ZJ. Evaluation of serum amino acid profiles' utility in non-small cell lung cancer detection in Polish population. Lung Cancer. 2016;100:71-76. doi:10.1016/j.lungcan.2016.04.008

34. Neurauter G, Grahmann AV, Klieber M, et al. Serum phenylalanine concentrations in patients with ovarian carcinoma correlate with concentrations of immune activation markers and of isoprostane-8. Cancer Lett. 2008;272(1):141-147. doi:10.1016/j.canlet.2008.07.002

35. Ploder M, Neurauter G, Spittler A, Schroecksnadel K, Roth E, Fuchs D. Serum phenylalanine in patients post trauma and with sepsis correlate to neopterin concentrations. Amino Acids. 2008;35 (2):303-307. doi:10.1007/s00726-007-0625-x

36. Lynch CJ, Adams SH. Branched-chain amino acids in metabolic signalling and insulin resistance. Nat Rev Endocrinol. 2014;10 (12):723-736. doi:10.1038/nrendo.2014.171

37. Jewell JL, Russell RC, Guan KL. Amino acid signalling upstream of mTOR. Nat Rev Mol Cell Biol. 2013;14(3):133-139. doi:10.1038/ nrm3522

38. Tato I, Bartrons R, Ventura F, Rosa JL. Amino acids activate mammalian target of rapamycin complex 2 (mTORC2) via PI3K/Akt signaling. J Biol Chem. 2011;286(8):6128-6142. doi:10.1074/jbc. M110.166991

39. Hattori A, Tsunoda M, Konuma T, et al. Cancer progression by reprogrammed BCAA metabolism in myeloid leukaemia. Nature. 2017;545(7655):500-504. doi:10.1038/nature22314 


\section{Publish your work in this journal}

Cancer Management and Research is an international, peer-reviewed open access journal focusing on cancer research and the optimal use of preventative and integrated treatment interventions to achieve improved outcomes, enhanced survival and quality of life for the cancer patient.

The manuscript management system is completely online and includes a very quick and fair peer-review system, which is all easy to use. Visit http://www.dovepress.com/testimonials.php to read real quotes from published authors. 